\title{
Development of Fingertip Tactile Sensing Chips for Humanoid Robots
}

\author{
Ravinder S. Dahiya ${ }^{1,2}$, G. Metta ${ }^{1,2}$, M. Valle ${ }^{2}$ \\ ${ }^{1}$ Robotics, Brain and Cognitive Sciences Department, \\ Italian Institute of Technology, Genoa, Italy, 16163 \\ ${ }^{2}$ University of Genoa, Genoa, Italy, 16145
}

\begin{abstract}
This paper presents the development of tactile sensing chips, for the fingertips of humanoid robots. In the first phase of development, piezoelectric polymer-MEA (microelectrode array) based test chips have been realized. Each chip comprises of 32 microelectrodes, epoxy-adhered with a thin piezoelectric polymer (PVDF-TrFE) film. The diameter of each microelectrode or 'taxel' (tactile element) is $500 \mu \mathrm{m}$ and the center to center distance between taxels is $1 \mathrm{~mm}$. The tactile sensing chips have been experimentally evaluated over a wide range $(0.02-4 \mathrm{~N}$ or $\sim 2$ gmf- $\mathbf{4 0 0}$ gmf) of dynamic normal forces and frequencies $(2 \mathrm{~Hz}-$ $5 \mathrm{KHz}$ ) by applying variable force with constant frequency in the first case and constant force with variable frequency in latter. The cross-talk, among adjacent taxels on the chips, is found to be approximately $20 \%$. Finally, the ability of the chips to identify object material on the basis of their hardness is also demonstrated.
\end{abstract}

\section{Keywords - Tactile Sensing, Tactile Sensing Arrays, MEA}

\section{INTRODUCTION}

The movement of robots from the structured environment of manufacturing plants to the daily life has led to the emergence of safety and interaction issues in robotics - more so, if a robot is made to work along with humans. Real-world objects also exhibit rich physical interaction behaviors, which depend on how heavy and hard the object is when hold; how their surface feels when touched; how they deform on contact and how they move when pushed etc. A part of the information about real world objects, e.g. shape, can be obtained by using vision cameras - which may be installed on a mobile platform, to overcome problems like occlusion [1]. But, the scope of vision based exploratory techniques is limited due to the fact that it is not always possible to take the cameras around the object. And even if it is possible, the presence of visual inaccuracies due to large distance between cameras and the object can make it difficult to explore and manipulate the given object. The methods to reduce such inaccuracies, e.g. eye-inhand configuration, too come at a cost - poor dexterity.

Intrinsic tri-axial or 6D force sensors, placed on the robot's wrist, have also been used to obtain information like contact location, both, for rigid and, to some extent, for soft contacts [2]. However, such methods are sensitive to the accuracy of force/torque sensor calibration and can provide erroneous information because of unmodeled dynamic forces. Further, compliance and inertia of manipulator may also interfere in such cases. Such problems can be avoided by equipping robot's

The work presented in this paper has been in part supported by the ROBOTCUB project (IST-2004-004370), funded by the European Commission through the Unit E5 -Cognitive Systems. hands with extrinsic touch sensors or tactile sensing arrays or a number of sensors distributed in a specific manner, thereby bringing the sensors closer to the contact points.

Clearly, problems like those mentioned above, make extrinsic touch sensing, or simply the tactile sensing, an essential component of a robot interacting with objects in a real world environment. Despite the important role, the research in tactile sensing has been lagging behind other sensory modalities e.g. vision and auditory sensing. This could partly be attributed to the distributed nature of tactile sensing and partly also to the unavailability of satisfactory tactile sensors. The pursuit of tactile sensing for robotic applications has resulted in the development of many 'benchtop' touch sensors exploring nearly all modes of transduction [3-6]. The tactile sensing technology largely remains unsatisfactory for humanoid robotics either because the developed sensors are single big size touch elements and are too big to be used without sacrificing the dexterity, or because they are slow, or fragile. Many innovative sensors could not find practical use due to the lack of system approach during the design stage [7].

Like sense of touch in humans, it is desirable to have tactile arrays, or a coordinated group of distributed tactile sensors, with density and spatial distribution of taxels (tactile elements) depending on the body site where the sensors are installed. In this sense, the sensors can be divided in two classes: one for the sites like fingertips that are involved in fine manipulation tasks and the other for the large area skin for safety/interaction etc. Whereas former requires a large number of taxels in a small space ( $\sim 1 \mathrm{~mm}$ spatial resolution) and fast response (of the order of few milliseconds); such a constraint can be relaxed for the latter [6]. The work presented here, focuses on the development of tactile sensing arrays for fingertips. Considering the limited available space on the robot finger $(\sim 1$ $\mathrm{cm} \times 1 \mathrm{~cm}$ ), miniaturization seems to be the solution for accommodating a large number of sensors. The same is also adopted in this work to develop tactile sensing arrays for the fingertips of humanoid robot iCub [8].

In past, miniaturization of tactile sensors has been achieved with two main approaches: MEMS based approach [9-11] and polymers based sensors realised on organic/inorganic substrate [12-15]. Polymers based touch sensors, realized on organic substrates, generally use pressure conductive rubber as transducer and are flexible. Due to higher spatial resolution $(\sim 2-4 \mathrm{~mm})$ and slower response, they are better suited to large area skin applications. As an example, in the $32 \times 32$ 


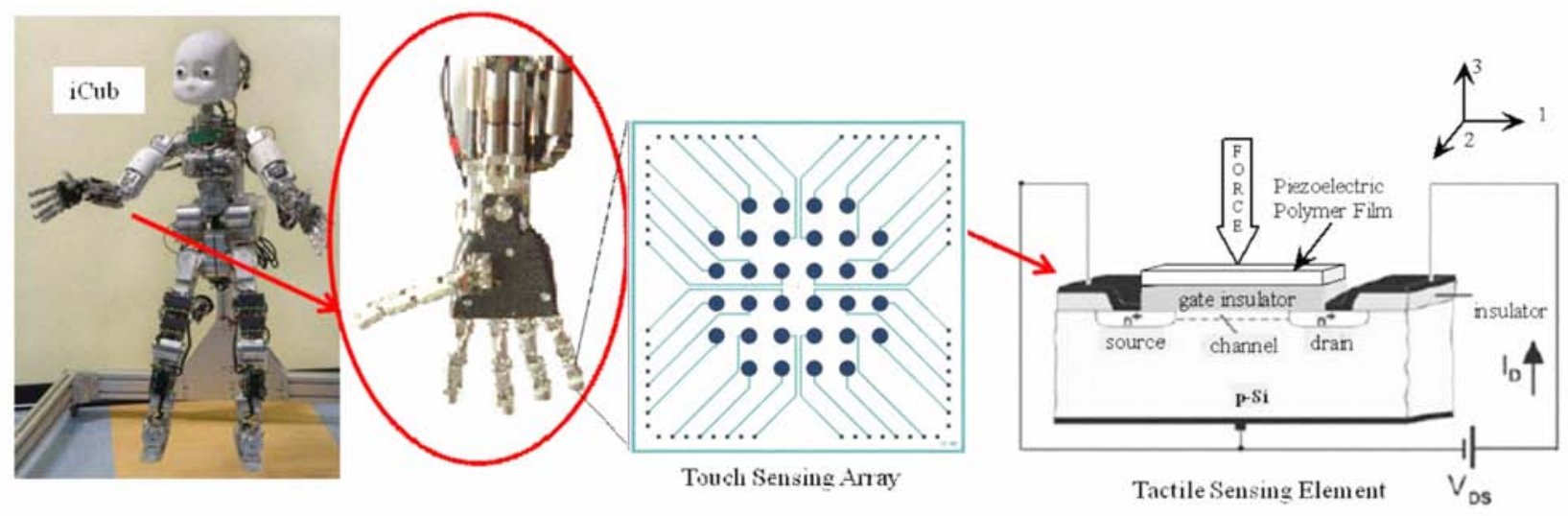

Fig. 1 Proposed Fingertip Tactile Sensing System

flexible tactile sensing array developed by Someya et al. [12] with organic FET approach, each taxel has a pitch of $2.54 \mathrm{~mm}$ and the response time of $30 \mathrm{~ms}$ (only of organic FETs). Overall response time is even higher because pressure sensitive rubber, used as transducer, has a response time of the order of hundreds of milli-seconds. These figures are quite high with respect to the one that can be obtained by standard silicon based IC (Integrated Circuit) technology. Thus, if such an array is placed on the fingertips then the requirements like high pitch and the fast response, would limit the number of taxels on the array.

MEMS based tactile sensing devices, generally use capacitive or piezoresistive mode of transduction. While piezoresistive devices offer higher linearity, the capacitive devices are an order of magnitude more sensitive. With the MEMS based approach, it is possible to get higher spatial resolution. A MEMS based approach has also been used for detecting the shear components of contact force, with a piezoresistive bridge arrangement on the sensing array [9]. But, MEMS based tactile sensing devices cannot withstand large forces/pressure due to their inherent fragile nature. Also, it is difficult to realize flexible tactile sensing arrays by the MEMS approach. A MEMS based flexible tactile sensing array has been reported in [10], where the silicon diaphragm has sensing pixel array on it and a pressure chamber beneath. The diaphragm is swollen like a balloon by the pressurized air, provided to the chamber through the hole and hence the stiffness of the diaphragm is controlled by the air pressure. In this way, a force in the range of $2.1 \mathrm{gmf}-17.6 \mathrm{gmf}(0.021 \mathrm{~N}-$ $0.176 \mathrm{~N}$ ) is reported to be controlled by pressure in the range of 5 - $64 \mathrm{KPa}$. The extra provision for air supply and its monitoring is cumbersome and such an arrangement is unsuitable for robots.

This work, presents the MEA based tactile sensing chips realized for the fingertips, during the first developmental phase, and the experimental results obtained from them. The tactile sensing chips use "smart materials" viz: piezoelectric polymer PVDF-TrFE (Polyvinylidene Fluoride - Trifluoroethylene), as transducer. Fig. 1 shows the proposed tactile sensing system. The tactile sensing chip consists of a 2-D array of 32 microelectrodes epoxy adhered with thin piezoelectric polymer film. Each microelectrode can be connected to the gate terminal of the FET devices, thereby acting as extended gate of the FET devices (external to the chip). The extended gate approach brings the sensor and conditioning electronics closer and hence the overall response is better than that of conventional approach - where the sensor and conditioning electronics are placed at a distance. While the piezoelectric polymer film as a transducer would improve the speed of response; the marriage of transducer and electronics will improve force resolution, spatial resolution, signal to noise ratio and also has potential of reducing the wiring complexity a key robotics problem.

\section{CONCEPT OF TACTILE SENSING ChiP}

\section{A. Concept}

A piezoelectric film working in the sensing mode generates a charge/voltage when mechanical force/stress is applied. This charge/voltage is proportional to the applied force/stress [16] and they are related as:

$$
Q_{t}=d_{33} \times F
$$

Where, $Q_{t}$ is the charge developed on a taxel due to applied force $F$ and $d_{33}$ is the piezoelectric constant. By connecting the piezoelectric polymer to the gate terminal of FET devices or by depositing the piezoelectric polymer directly on the gate area of FET devices, such a charge/voltage of the piezoelectric polymer can be used to modulate the charge in the induced channel of FET, which is then amplified by the FET and further processed by an electronic circuitry. A somewhat similar approach was used by Swartz et al. [17] for the development of ultrasonic sensors and by Kolesar et al. [15] for the touch sensors. In both cases, the extended gate FET devices epoxy-adhered with PVDF film were used. In present work, the PVDF-TrFE polymer films are used, as unlike PVDF, they do not need mechanical stretching [16] - a standard step while making PVDF polymer films. This is a significant advantage of PVDF-TrFE as it allows the spin coating of polymer directly on to silicon wafer. In addition, PVDF-TrFE has nearly same piezoelectric constant as that of PVDF, has lesser losses and generates approximately the same charge/voltage output for the same force input. 


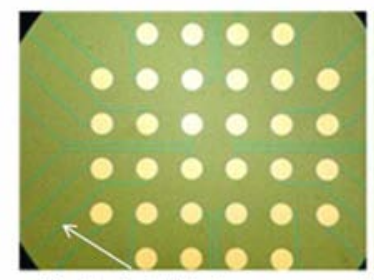

Passivated Metal
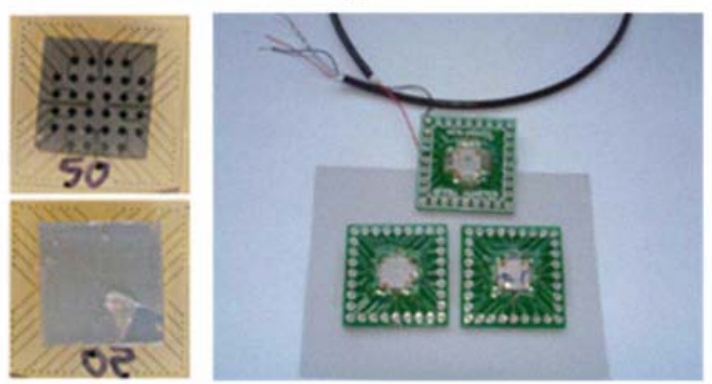

(b)

2 (a) 32 taxel MEA test chip (left) with enlarged view of a ht); (b) Front and backsides of MEA test chip epoxy-adhered wi1 PVDF-TrFE film (left); Packaged MEA chips (right).

\section{B. Tactile Sensing Chip}

As a first step towards realizing tactile sensing system, two dimensional, 32 taxel, MEAs based test chips are designed and fabricated on quartz wafer. Detailed design and fabrication process are explained elsewhere [18]; however, the key points are briefly given here. The overall size of MEA chip is $1 \mathrm{~cm} \mathrm{x}$ $1 \mathrm{~cm}$ and each taxel on the chip has a diameter of $500 \mu \mathrm{m}$. To get a spatial resolution comparable to that of human tactile sensing $(\sim 1 \mathrm{~mm})$, the center to center distance between the taxels is kept as $1 \mathrm{~mm}$. Here, quartz wafer is used instead of silicon to minimize the noise. The fabricated MEA test chip and enlarged view of one of the taxels are shown in Fig. 2(a). Each sensing element on the MEA test chip can be used as extended gate of the FET devices, which are external to the chip.

The MEA test chips were epoxy-adhered with one side metalized piezoelectric polymer (70:30, PVDF-TrFE) thin films of $25 \mu \mathrm{m}, 50 \mu \mathrm{m}$ and $100 \mu \mathrm{m}$. Polymer films with different thickness are used to study the effect of thickness on the response of a taxel. The front and backsides of a MEA test chip with a $50 \mu \mathrm{m}$ polymer film and the chip on package are shown in Fig 2(b). On the package, only 28 terminals (out of 32) are gold bonded to the pads of MEA as all corners are gold bonded to the upper metal layer of the piezoelectric polymer film. Thus, corners of the package, all together, serve as one of the terminals of charge/voltage source (generated by the applied force).

\section{EXPERIMENT SET UP}

\section{A. Mechanical Arrangement}

The experimental set up developed for testing the tactile sensing chips is shown in Fig. 3. The TIRA shaker on the set up can apply random dynamic forces up to $18 \mathrm{~N}$ with frequency in the range $2 \mathrm{~Hz}-18 \mathrm{KHz}$. The shaker can be fixed at any angle $\theta\left(0<\theta<90^{\circ}\right)$ by adjusting turnbuckles and hence the direction of applied forces can also be changed. The force generated by the shaker is measured by piezoelectric load cell (PCB Piezotronics), which can move along the z-axis. The load cell has a sensitivity of $112.41 \mathrm{mV} / \mathrm{kN}$ and the measurement range of $0.04448 \mathrm{kN}$ (both in compression and tension). To apply force on a single taxel at a time and also on many taxels simultaneously, special probes having diameter of $1 \mathrm{~mm}$ are developed. The diameter of the probe is slightly higher than that of the taxel, to ensure that the whole taxel is pressed during testing. These probes are attached to the load cell with a screw. With a manual $x-y$ positioning system the probe is aligned with the taxel under observation. The MEA test chip is placed on the shaker, as shown in Fig. 3 and then the load cell is brought in contact with it with help of the z-axis positioning motor. The vibration generator/shaker, piezoelectric load cell and the zaxis positioning motor and the output of taxel are all measured and controlled with LABVIEW through NI-DAQ device (PCI6221) which can acquire and control the data from 16 analog outputs, 24 digital $\mathrm{I} / \mathrm{O}$ and 2 analog inputs.

\section{B. Electrical Arrangement}

To measure the output of the taxels on the MEA test chip, both, charge and voltage amplifiers were designed on two separate PCBs. Both charge and voltage amplifiers were designed for a frequency range of $2 \mathrm{~Hz}-18 \mathrm{KHz}$. The independence of output from the cable capacitances and the minimization of the charge leakage through the stray capacitance around the sensor, are the advantages of the charge amplifier. The arrangement of the charge amplifier along with an approximate equivalent model of a taxel, is shown in Fig. 4.

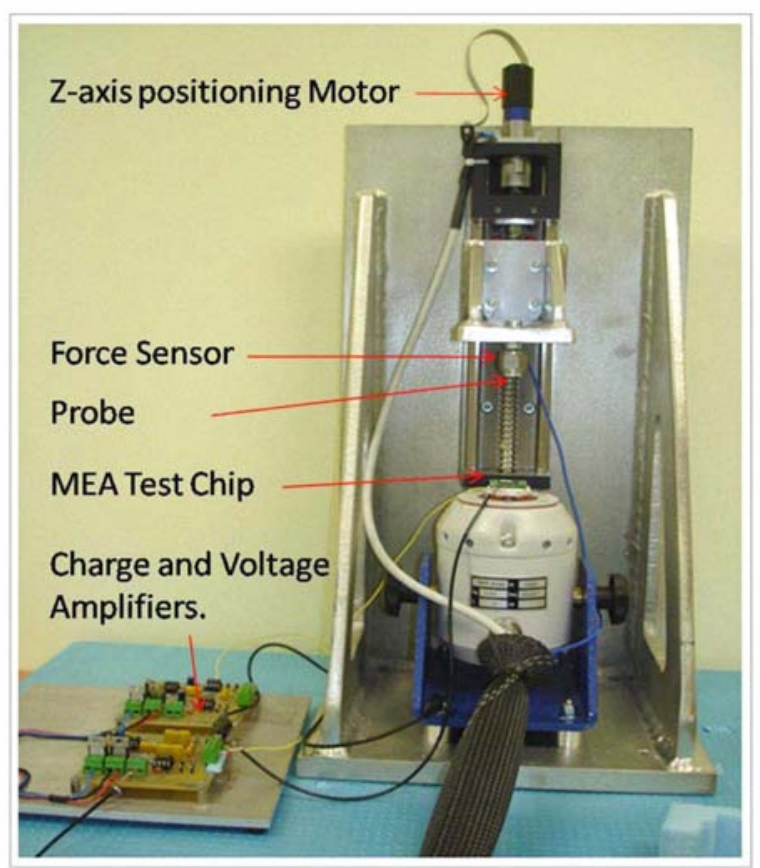

Fig. 3 Experiment set up for testing the tactile sensing chips. 


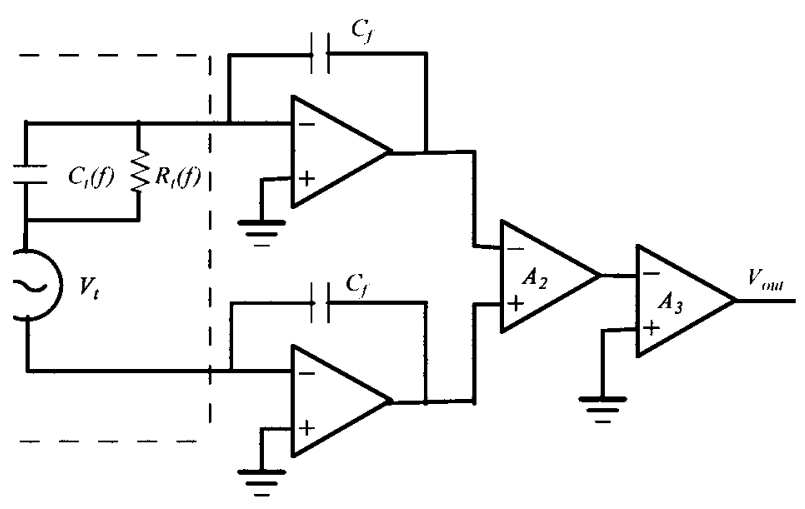

4: Approximate equivalent model of a taxel (inside the dotted box 1 charge amplifier.

\section{EXPERIMENTAL RESULTS}

The performance of MEA based test chips (with $25 \mu \mathrm{m}, 50$ $\mu \mathrm{m}$ polymer films) is evaluated in variable force - constant frequency and constant force - variable frequency configurations, under ambient conditions. In the variable force - constant frequency mode, a normal sinusoidal $15 \mathrm{~Hz}$ force, in the range $0.02 \mathrm{~N}-4 \mathrm{~N}(\sim 2 \mathrm{gmf}-400 \mathrm{gmf})$, was applied on the taxels. In these experiments the taxels are pre stressed by a $4 \mathrm{~N}$ force. The resulting plots between average taxel output and the force input are shown in Fig. 5. These plots relate average maximum values of the sinusoidal output of three taxels located at the centre of chips, with corresponding maximum values of the applied sinusoidal forces. The response of each of the three taxels tested in this case was within approximately $6 \%$ of the average output shown in Fig. 5. Such a variation among outputs of different taxel could be due to the nonuniform thickness of polymer or due to slight misalignment of probe and taxel. Besides others, the uniform deposition of a thin polymer film is a challenging task for the tactile sensing arrays presented in this work. A higher variation is also observed in the response of the taxels located on the corners of chips. This could be due to the fact that top metal layer was gold bonded at all four corners of the chip, which requires high temperature $\left(>100^{\circ} \mathrm{C}\right)$ that might have altered the properties of polymer on the corner taxels. To avoid any damage to the chip, forces higher than $4 \mathrm{~N}$ are not applied at this stage. The approximate expression of output voltage at the terminals of each taxel, $V_{l}$ and at the output terminals of charge amplifier, $V_{\text {out }}$, are respectively given by:

$$
\begin{aligned}
& V_{t} \approx \frac{d_{33} \times F \times A_{t}}{C_{t}(f) \times A_{p}} \\
& V_{\text {out }} \approx \frac{2 \times C_{t}(f) \times A_{2} \times A_{3} \times V_{t}}{C_{f}} \\
& \frac{V_{\text {out }}}{F} \approx \frac{2 \times d_{33} \times A_{2} \times A_{3} \times A_{t}}{C_{f} \times A_{p}}
\end{aligned}
$$

Where, $A_{t}$ is the area of each taxel; $A_{p}$ is the area of probe (or the area on which force is applied); $C_{t}(f)$ is the capacitance

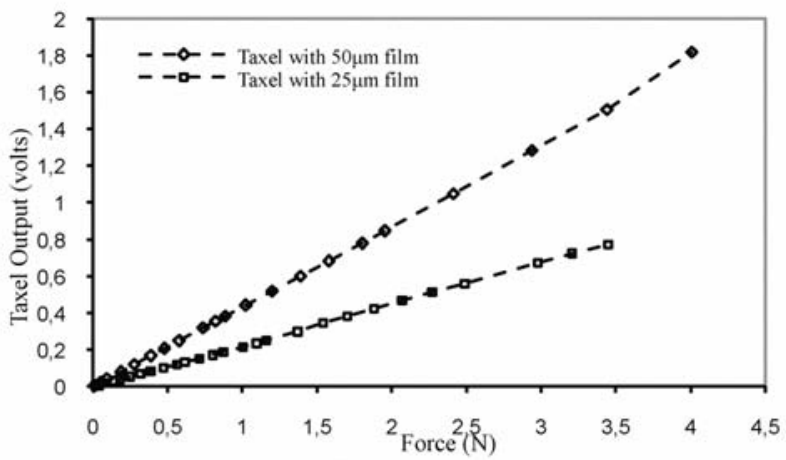

Fig. 5 Average maximum of sinusoidal taxel output versus maximum value of sinusoidal input force.

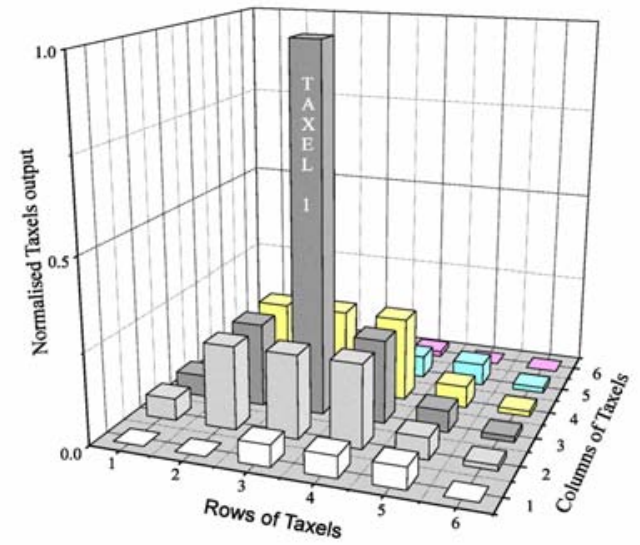

ig. 6: Normalized output of various taxels on the array with respect to the utput of taxel (marked as 'Taxel 1') on which force is applied. lormalized valued were obtained by dividing output of various taxels with lat marked as 'Taxel 1'.

of polymer on each taxel; $C_{f}$ is the capacitance in the feedback path of first stage of amplifier and $A_{2}, A_{3}$ are the gains of second and third stage of the amplifier respectively. From (4), it can be noticed that at a particular frequency, the voltage output of a taxel is linearly dependent on the input force. The plots in Fig. 5 also follow this trend over the tested range of forces.

The response of taxels on both chips with $50 \mu \mathrm{m}$ and $25 \mu \mathrm{m}$ thick polymer films is further investigated for "cross-talk" as spatial resolution also depends on it. The higher the cross talk, the lower will be the spatial resolution. To investigate cross talk, the force is applied on a particular taxel and the voltage output from the adjacent taxels is observed. Fig. 6 shows the output of various taxels, with respect to the output of the particular taxel (say, taxel no. 1) at the center of chip, on which force is applied. With the taxel outputs noted in this way, the crosstalk in both the chips is found to be approximately $20 \%$. Although the taxels are independent entities under the polymer film (MEA side of the film); they are all connected by a uniform thin metal film on the top. In other words, taxels are still mechanically connected, which is the primary reason for the presence of the cross talk in both the chips. The parasitic capacitance between adjacent elements also contributes to 

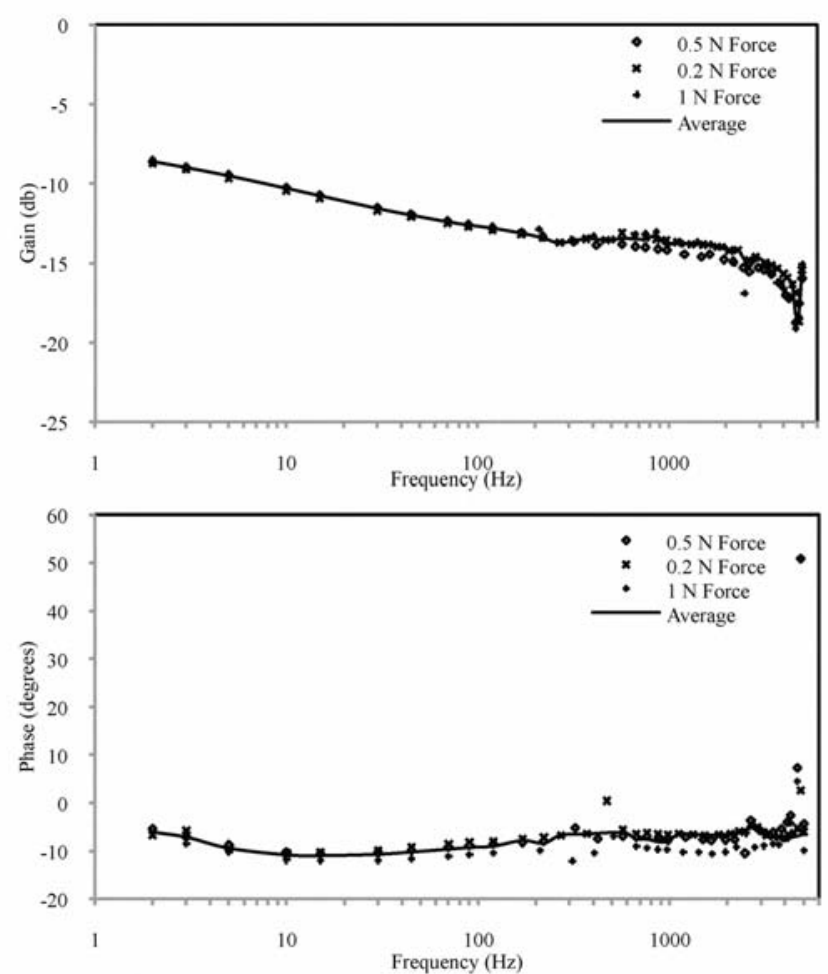

Fig. 7: Gain (top) and phase (bottom) plots of MEA with $50 \mu \mathrm{m}$ polymer film at three different applied forces, viz: $0.2 \mathrm{~N}, 0.5 \mathrm{~N}$ and $1 \mathrm{~N}$. Solid line corresponds to the average values of the three forces.

cross-talk. The problem of cross-talk can be reduced by patterning the metal layer on the top side of polymer film and thus reducing the taxels dependence on each other. This will be implemented in the next phase when tactile sensing chip will be redesigned for better integration with the robot.

In the constant force - variable frequency mode, the chip is evaluated in the frequency range of $2 \mathrm{~Hz}-5 \mathrm{KHz}$ for three different forces, viz: $0.2 \mathrm{~N}, 0.5 \mathrm{~N}$ and $1 \mathrm{~N}$. The gain $\left(=V_{\text {out }} / F\right)$ and phase plots are shown in Fig. 7. Using the actual values of $A_{2}=5, A_{3}=4, d_{33}=24 \times 10^{-12}$ (at $\left.1 \mathrm{KHz}\right), A_{\mathrm{t}}=0.196 \times 10^{-6} \mathrm{~m}^{2}$, and $A_{\mathrm{p}}=0.78 \times 10^{-6} \mathrm{~m}^{2}$, and $C_{\mathrm{f}}=820 \mathrm{pF}$ in (4), the gain in $\mathrm{db}$ (at $1 \mathrm{KHz}$ ) is -16.5 , which closely matches the experimental values shown in Fig 7. The gain has a drooping characteristic over the entire range of frequency for which the chip has been evaluated. It is a general practice to use a constant value of the piezoelectric polymer capacitance (at $1 \mathrm{KHz}$ ). But, in case of piezoelectric polymers this is not true, as can be seen from Fig. 8, where the capacitance, $C_{\mathrm{t}}(f)$ and resistance, $R_{\mathrm{t}}(f)$ of a taxel are plotted against the frequency. These values of $C_{\mathrm{t}}(f)$ and $R_{\mathrm{t}}(f)$ were measured with an impedance analyzer. In addition, the value of piezoelectric constant also depends on the frequency. Thus, the drooping gain characteristic can be attributed to the frequency dependent constants. The phase plot shows more or less a constant value of phase in the said tested frequency range. The lower cut off frequency is not seen in these plots, because the charge amplifier is designed to have a lower cut-off frequency less than $2 \mathrm{~Hz}$. In Fig. 7, the values of gain and phase deviate significantly from the general trend at around $500 \mathrm{~Hz}, 2600 \mathrm{~Hz}$ and $4800 \mathrm{~Hz}$. This is due to

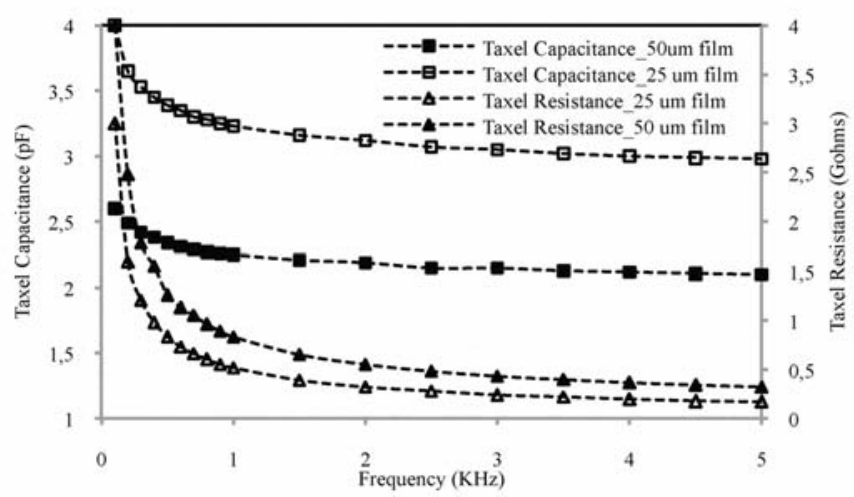

Fig. 8: Variation of the capacitance, $C_{t}$ and resistance, $R_{t}$ of taxels having with frequency.

mechanical resonance of the shaker at these points. Such points have been neglected while calculating the average values.

The tactile sensing chips have been further evaluated for their ability to differentiate objects on the basis of hardness. For this purpose, probes with $1 \mathrm{~mm}$ diameter, made from brass (density $\sim 8500 \mathrm{Kg} / \mathrm{m}^{3}$ ), polycarbonate plastic (density $\sim 1200$ $\mathrm{Kg} / \mathrm{m}^{3}$ ) and wood (density $\sim 500 \mathrm{Kg} / \mathrm{m}^{3}$ ), are used to apply a force with same magnitude and the outputs of a taxels are noted. The observed outputs from taxels with both $50 \mu \mathrm{m}$ and $25 \mu \mathrm{m}$ thick polymer films are shown in Fig. 9. The outputs of taxels from both chips are noted by applying forces of $0.2 \mathrm{~N}$ and $0.4 \mathrm{~N}$ at various frequencies between $5-70 \mathrm{~Hz}$. Higher forces are avoided to prevent the damage of the probe, especially the one made of wood. With the exception at $5 \mathrm{~Hz}$ in the case of taxel with $50 \mu \mathrm{m}$ film, the output with brass probe is higher that from polycarbonate plastic and wooden probe. In the case of taxel with $25 \mu \mathrm{m}$ film, the difference between outputs from brass probe and polycarbonate plastic/wooden probe is higher than that of taxel with $50 \mu \mathrm{m}$ film. The difference between outputs obtained from wooden probe and polycarbonate plastic probe is not high due to small difference between their densities. The object identification based on hardness is more distinct in frequency domain, at frequencies close to the resonant frequency (approximately $24 \mathrm{MHz}$ and 48 $\mathrm{MHz}$ for $50 \mu \mathrm{m}$ and $25 \mu \mathrm{m}$ polymer films) of the polymer film [19]. To further quantify this ability of differentiating the objects on the basis of their hardness, more taxels on each array, will be tested in future.

\section{CONCLUSION}

An approach for the development of tactile sensing chips for humanoid robots is presented along with the first prototype test chips. The MEA based chips have been experimentally evaluated by applying dynamic normal forces up to $4 \mathrm{~N}$. The output-input relation is linear and cross talk is $\sim 20 \%$. The gain and phase plots obtained in the frequency range $(2 \mathrm{~Hz}-5$ $\mathrm{KHz}$ ) have been presented. Further the ability of chips to differentiate objects on the basis of their hardness has been demonstrated.

The lack of flexibility is the major disadvantage of tactile sensing arrays based on standard silicon IC technology. Using soft and compliant polymer as substrate, having mechanically integrated, but, otherwise distinct and stiff islands of the tactile 


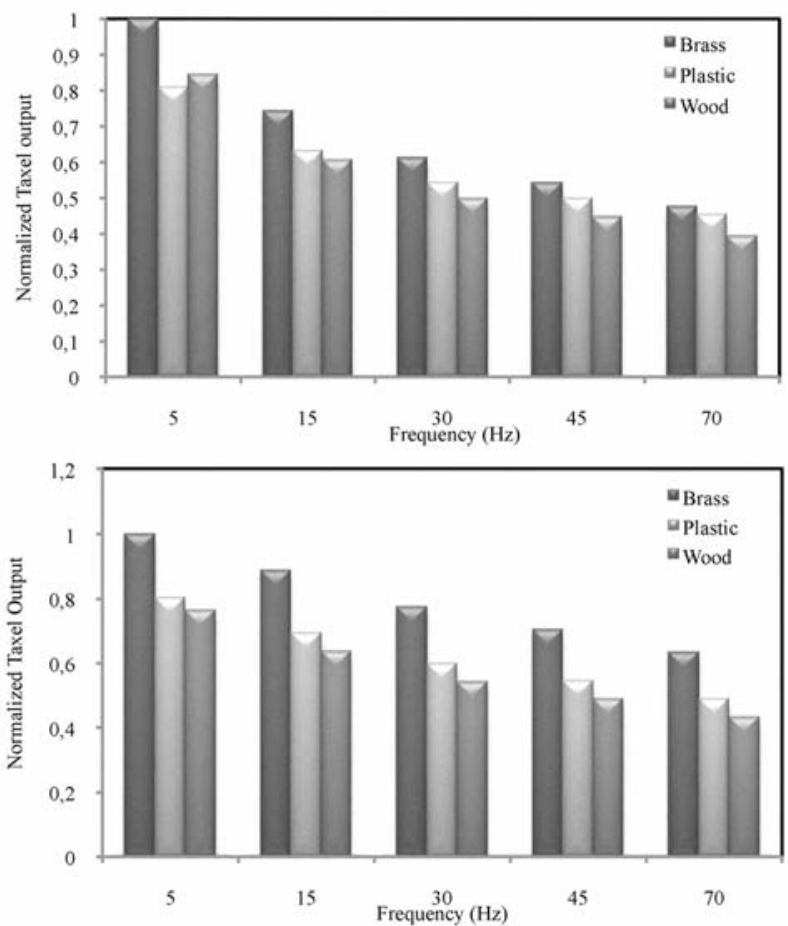

Fig. 8: Output voltage of the taxel from MEA with $50 \mu \mathrm{m}$ film (top) and 25 $\mu \mathrm{m}$ film (bottom) when it is pressed by probes made of brass, plastic and wood.

sensing arrays, connected to each other by flexible and stretchable metal interconnects, could be a plausible solution for having a conformable electronic surface. Another possible trade off is to cover the chip with a thick and protective layer of silicone. Due to low thermal conductivity, such a layer would be helpful in reducing the effect of ambient temperature variations also - which otherwise introduces noise in the output during measurement of forces. However, a careful study is needed as such materials suffer from creep, hysteresis and in practice work as low pass filters [20].

Fabrication of tactile sensing chips with on-chip simple electronic circuitry will help in better exploiting the benefits offered by the approach presented in this work. Distributed nature of tactile sensing calls for large number of tactile sensors spread over the body, resulting in a large amount of data. In such a situation, on-chip tactile information processing, like on-chip slip detection [21], can help in optimally using the limited computational resources of robots [7]. Accommodating on-chip electronic circuitry requires study of challenging issues like those related to integration of the sensors and electronics and also their coupling. For easier implementation and to minimize overall cost of manufacturing, the sensing technology should also be compatible with standard CMOS technology. Such issues will require an attention in future. Piezoelectric polymers are anisotropic in nature - which makes them suitable for detecting the direction of force. This property can also be exploited in future to use the tactile sensing chips for detecting and measuring the forces applied in shear direction. PVDFTrFE responds to variations in temperature also and this can be used for the detection of temperatures. Such properties can be exploited to extend the multifunctionality of tactile sensing chips, so as to detect and measure not only forces and hardness but also the temperature. Finally, it will be interesting to see how the tactile sensing chips perform when they are integrated on the fingertips of the robot to explore real world objects.

\section{REFERENCES}

[1] A. S. Ogale, C. Fermuller, and Y. Aloimonos, "Motion Segmentation Using Occlusions," IEEE Transactions on Pattern Analysis and Machine Intellingence, vol. 27, pp. 988-992, 2005.

[2] A. Bicchi, K. Salisbury, and D. L. Brock, "Contact sensing from force and torque measurements," The International Journal of Robotics Research, vol. 12, pp. 249-262, 1993.

[3] R. D. Howe, "Tactile Sensing and Control of Robotics Manipulation," Journal of Advanced Robotics, vol. 8, pp. 245-261, 1994.

[4] M. H. Lee and H. R. Nicholls, "Tactile Sensing for Mechatronics - A State of the Art Survey," Mechatronics, vol. 9, pp. 1-31, 1999.

[5] P. Dario and D. de Rossi, "Tactile Sensors and Gripping Challenge," IEEE Spectrum, vol. 22, pp. 46-52, 1985.

[6] R. S. Dahiya and M. Valle, "Tactile Sensing for Robots," in Sensors, Focus on Tactile, Force and Stress Sensors, V. Kordic, Ed. Vienna, Austria: ITech Education and Publishing, 2008

[7] R. S. Dahiya, M. Valle, and G. Metta, "System Approach-A paradigm for Robotic Tactile Sensing," in The 10th IEEE International Workshop on Advanced Motion Control, Trento, Italy, March, 2008, pp. 110-115.

[8] G. Sandini, G. Metta, and D. Vernon, " RobotCub: An Open Framework for Research in Embodied Cognition " in IEEE-RAS/RSJ Int. Conf. on Humanoid Robots, Humanoids 2004, Santa Monica, CA, USA., 2004.

[9] B. J. Kane, M. R. Cutkosky, and G. T. A. Kovacs, "A Traction Stress Sensor Array for Use in High-Resolution Robotic Tactile Imaging," Journal of Microelectromechanical Systems, vol. 9, pp. 425-434, 2000.

[10] H. Takao, K. Sawada, and M. Ishida, "Monolithic Silicon Smart Tactile Image Sensor With Integrated Strain Sensor Array on Pneumatically Swollen Single-Diaphragm Structure," IEEE Transactions on Electron Devices, vol. 53, pp. 1250-1259, 2006.

[11] K. Suzuki, K. Najafi, and K. D. Wise, "A 1024-Element HighPerformance Silicon Tactile Imager," IEEE Transactions on Electron Devices, vol. 37, pp. 1852-1860, 1990.

[12] T. Someya, T. Sekitani, S. Iba, Y. Kato, H. Kawaguchi, and T. Sakurai, " A Large-Area, Flexible Pressure Sensor Matrix with Organic Field-Effect Transistors for Artificial Skin Applications," PNAS, vol. 101, pp. 9966-9970, 2004

[13] J. Engel, J. Chen, Z. Fan, and C. Liu, "Polymer Micromachined Multimodal Tactile Sensors," Sensors and Actuators A, vol. 117, pp. 50-61, 2005.

[14] M. Shimojo, A. Namiki, M. Ishikawa, R. Makino, and K. Mabuchi, "A Tactile Sensor Sheet Using Pressure Conductive Rubber With ElectricalWires Stitched Method," IEEE Sensors Journal, vol. 4, pp. 589-596, 2004.

[15] E. S. Kolesar and C. S. Dyson, "Object Imaging with a Piezoelectric Robotic Tactile Sensor," Journal of Microelectromechanical Systems, vol. 4, pp. 87-96, 1995.

[16] H. S. Nalwa, "Ferroelectric Polymers: Chemistry, Physics and Applications," Mercel Dekker Inc., 1995.

[17] R. G. Swartz and J. D. Plummer, "Integrated silicon-PVF2 acoustic transducer arrays," IEEE transactions on Electron Devices, vol. 26, pp. 1920$32,1979$.

[18] R. S. Dahiya, M. Valle, G. Metta, L. Lorenzelli, and C. Collini, "Tactile Sensor Arrays for Humanoid Robot," in IEEE PRIME'07, The 3rd International Conference on $P h D$ Research in Microelectronics and Electronics, Bordeaux, France, July, 2007, pp. 201-204.

[19] R. S. Dahiya, M. Valle, G. Metta, and L. Lorenzelli, "POSFET Based Tactile Sensor Arrays," in IEEE ICECS'07, The 14th Int. Conf. on Electronics, Circuits and Systems, Marrakech, Morocco, Dec, 2007, pp. 1075-1078.

[20] M. Shimojo, "Mechanical Filtering Effect of Elastic Cover for Tactile Sensor," IEEE Trans. on Robotics And Autom., vol. 13, pp. 128-132, 1997.

[21] R. Maldonado-López, F. Vidal-Verdú, G. Liñán, E. Roca, and A. Rodríguez-Vázquez, "Early Slip Detection with a tactile sensor based on retina," Analog Integr. Circ. and Signal Processing, vol. 53, pp. 97-108, 2007. 